\title{
RELATIONSHIP BETWEEN IDEALS OF BCI-ALGEBRAS AND ORDER IDEALS OF ITS ADJOINT SEMIGROUP
}

\author{
MICHIRO KONDO
}

(Received 4 October 2000 and in revised form 6 February 2001)

\begin{abstract}
We consider the relationship between ideals of a BCI-algebra and order ideals of its adjoint semigroup. We show that (1) if $I$ is an ideal, then $I=M^{-1}(M(I)),(2) M\left(M^{-1}(J)\right)$ is the order ideal generated by $J \cap R(X)$, (3) if $X$ is a BCK-algebra, then $J=M\left(M^{-1}(J)\right)$ for any order ideal $J$ of $X$, thus, for each BCK-algebra $X$ there is a one-to-one correspondence between the set $\mathscr{I}(X)$ of all ideals of $X$ and the set $\mathcal{O}(X)$ of all order ideals of it, and (4) the order $M\left(M^{-1}(J)\right)$ is an order ideal if and only if $M^{-1}(J)$ is an ideal. These results are the generalization of those denoted by Huang and Wang (1995) and Li (1999). We can answer the open problem of Li affirmatively.
\end{abstract}

2000 Mathematics Subject Classification. 06F35, 03G25.

1. Introduction. In [1, 2, 4], the relationship between filters of a BCK-algebra and order ideals of its adjoint semigroup is considered. There is a gap in the proofs of the results obtained there. For example, [2, Theorem 2.8] and [4, Theorem 2.3], where it is proved for a map $I \rightarrow M(I)$ to be onto, is not correct. They only show that if $I$ is a filter then $M(I)$ is an order ideal and that if $J$ is an order ideal then $M^{-1}(J)$ is a filter. In order to fill the gap of the proof and to develop the theory of adjoint semigroups of BCI-algebras, we consider ideals instead of filters and show more results about order ideals. As a particular case, we give an affirmative solution to the problem left open in [4].

First of all, we define BCI-algebras.

Let $\langle X ; *, 0\rangle$ be an algebraic structure of type $\langle 2,0\rangle$. We call it a BCI-algebra when it satisfies the conditions: for any $x, y, z \in X$,

(1) $(x * y) *(x * z) \leq z * y$,

(2) $x *(x * y) \leq y$,

(3) $x * x=0$,

(4) if $x * y=y * x=0$ then $x=y$.

The relation " $\leq$ " is defined as follows:

$$
x \leq y \Leftrightarrow x * y=0 .
$$

It is easy to show that $\langle X ; \leq\rangle$ is a partially-ordered set and the following proposition holds.

Proposition 1.1. Let $X$ be a BCI-algebra. For any $x, y, z \in X$,

(a) $x * 0=x$,

(b) $0 *(x * y)=(0 * x) *(0 * y)$, 
(c) $(x * y) * z=(x * z) * y$,

(d) $(x * z) *(y * z) \leq x * y$,

(e) $x \leq y$ implies $x * z \leq y * z$,

(f) $x \leq y$ implies $z * x \leq z * y$.

Moreover, a BCI-algebra is called a BCK-algebra if it satisfies the condition

(5) $0 * x=0$.

Any subset $I$ of $X$ is said to be an ideal if

(I1) $0 \in I$,

(I2) $x * y, y \in I \Rightarrow x \in I$.

Let $X$ be any BCI-algebra. For an element $a \in X$, we define a map $a^{-1}$ from $X$ into itself by

$$
(x) a^{-1}=x * a .
$$

The map $a^{-1}$ is called a right map in [3]. We also define $M(X)$ and $R(X)$ for any BCI-algebra $X$ as follows:

$$
M(X)=\left\{a_{1}^{-1} a_{2}^{-1} \cdots a_{n}^{-1} \mid a_{1}, a_{2}, \ldots, a_{n} \in X\right\}, \quad R(X)=\left\{a^{-1} \mid a \in X\right\},
$$

where $a_{1}^{-1} a_{2}^{-1} \cdots a_{n}^{-1}$ is a map defined by

$$
(x) a_{1}^{-1} a_{2}^{-1} \cdots a_{n}^{-1}=\left(\cdots\left(\left(x * a_{1}\right) * a_{2}\right) \cdots\right) * a_{n} .
$$

We call $M(X)$ an adjoint semigroup of $X$. It follows from the properties of BCIalgebras that $M(X)$ is a commutative monoid with unit $0^{-1}$. We introduce a relation $\subseteq$ on $M(X)$ :

$$
\begin{gathered}
b_{1}^{-1} b_{2}^{-1} \cdots b_{m}^{-1} \sqsubseteq a_{1}^{-1} a_{2}^{-1} \cdots a_{n}^{-1} \\
\Uparrow \\
\left(\cdots\left(\left(u * a_{1}\right) * a_{2}\right) \cdots\right) * a_{n} \leq\left(\cdots\left(\left(u * b_{1}\right) * b_{2}\right) \cdots\right) * b_{m} \quad(u \in X) .
\end{gathered}
$$

It is obvious that $\langle M(X)$, $\subseteq\rangle$ is a partially ordered set. We note that for any element $a, b \in X$,

$$
a^{-1} \sqsubseteq b^{-1} \Longleftrightarrow a \leq b .
$$

In fact, if $a^{-1} \sqsubseteq b^{-1}$ then $u * b \leq u * a$ for every $u \in X$. If we take $u=a$, then $a * b=0$ and thus $a \leq b$. Conversely, if we suppose $a \leq b$ then, since $(u * b) *(u * a) \leq a * b=0$ for any $u \in X$, we have $u * b \leq u * a$. This means that $a^{-1} \sqsubseteq b^{-1}$.

For any subset $S$ of $X$, we define

$$
M(S)=\left\{a_{1}^{-1} a_{2}^{-1} \cdots a_{n}^{-1} \mid a_{i} \in S(1 \leq i \leq n)\right\} .
$$

A subsemigroup $J$ of $M(X)$ is called an order ideal if it satisfies the condition

$$
\forall x \in J \text { and } y \in R(X) \text {, if } y \sqsubseteq x \text { then } y \in J .
$$

Proposition 1.2. Let $X$ be a BCI-algebra. Then the following statements are equivalent:

(a) $X: B C K$-algebra. 
(b) $0^{-1}$ is the smallest element in $M(X)$.

(c) For any element $x^{-1}, y^{-1} \in R(X), x^{-1} \sqsubseteq x^{-1} y^{-1}$.

Proof. (a) $\Rightarrow$ (b). For every element $a_{1}^{-1} a_{2}^{-1} \cdots a_{n}^{-1} \in M(X)\left(a_{i} \in X\right)$ and $u \in X$, since $X$ is a BCK-algebra, we have $\left(\left(\cdots\left(u * a_{1}\right) * \cdots\right) * a_{n}\right) *(u * 0)=\left(\left(\cdots\left(0 * a_{1}\right) * \cdots\right) *\right.$ $\left.a_{n}\right)=0$. This implies $\left(\cdots\left(u * a_{1}\right) * \cdots\right) * a_{n} \leq u * 0$ for any element $u \in X$. Thus we get $0^{-1} \sqsubseteq a_{1}^{-1} \cdots a_{n}^{-1}$ and $0^{-1}$ is the smallest element of $M(X)$.

(b) $\Rightarrow$ (c). Suppose that $0^{-1}$ is the smallest element of $M(X)$. Since $0^{-1} \sqsubseteq y^{-1}$ and the operation of $M(X)$ preserves the order $\sqsubseteq$, we have $x^{-1}=x^{-1} 0^{-1} \sqsubseteq x^{-1} y^{-1}$.

(c) $\Rightarrow$ (a). If we take $x^{-1}=0^{-1}$ then we have $0^{-1} \sqsubseteq y^{-1}$ and hence $0 \leq y$ for any $y \in X$. This means that $X$ is a BCK-algebra.

2. Basic properties of ideals and order ideals. In this section, we consider the basic properties of ideals and order ideals. Moreover, we investigate relations between ideals and order ideals. First of all we have the following result.

Proposition 2.1. If I is an order ideal, then $M(I)$ is an order ideal.

Proof. It is obvious that $M(I)$ is a subsemigroup of $M(X)$. Suppose $x \in M(I), y \in$ $R(X)$, and $y \sqsubseteq x$. There are elements $a_{1}, \ldots, a_{n} \in I$ and $b \in X$ such that $x=a_{1}^{-1} \cdots a_{n}^{-1}$ and $y=b^{-1}$. Since $y \subseteq x$, we have, for any $u \in X$,

$$
\left(\cdots\left(u * a_{1}\right) * \cdots\right) * a_{n} \leq u * b .
$$

Especially, if we take $u=b$, then

$$
\left(\cdots\left(b * a_{1}\right) * \cdots\right) * a_{n}=0 \in I .
$$

Since $I$ is the ideal and $a_{i} \in I$, we have $b \in I$ and hence $y=b^{-1} \in M(I)$.

Proposition 2.2. For any subsets $S, S^{\prime} \subseteq X$, if $S \neq S^{\prime}$ then $M(S) \neq M\left(S^{\prime}\right)$, that is, $M$ is an injection.

Proof. Suppose that $S \neq S^{\prime}$ for subsets $S, S^{\prime}$ of $X$. There exists an element $a \in X$ such that $a \in S$ but $a \notin S^{\prime}$. Since $a^{-1} \in M(S)$ and $a^{-1} \notin M\left(S^{\prime}\right)$, we get $M(S) \neq M\left(S^{\prime}\right)$. Thus the map $M$ is an injection.

We define a map $M^{-1}$ by

$$
M^{-1}(T)=\left\{x \mid x^{-1} \in T\right\}
$$

for every $T \subseteq M(X)$. It is clear that $M^{-1}(T)=M^{-1}(T \cap R(X))$.

Proposition 2.3. If $J$ is an order ideal, then $M^{-1}(J)$ is an ideal.

Proof. Since $J$ is an order ideal, it is of course a subsemigroup of $M(X)$ and hence $0^{-1} \in J$, that is, $0 \in M^{-1}(J)$.

We assume that $x, y * x \in M^{-1}(J)$ for $x, y \in X$. It follows from the definition of $M$ that $x^{-1}(y * x)^{-1} \in J$. On the other hand, we have $y^{-1} \sqsubseteq x^{-1}(y * x)^{-1}$ by the property of BCI-algebras. This yields that $y^{-1} \in J$ and hence that $y \in M^{-1}(J)$. 
From the above we have the following relations about ideals and order ideals:

$$
\begin{gathered}
I: \text { ideal } \Longrightarrow M(I) \text { : order ideal } \Longrightarrow M^{-1}(M(I)) \text { : ideal, } \\
J \text { : order ideal } \Longrightarrow M^{-1}(I) \text { : ideal } \Longrightarrow M\left(M^{-1}(J)\right) \text { : order ideal. }
\end{gathered}
$$

It is natural to ask whether $I$ is identical with $M^{-1}(M(I))$ or so is $J$ with $M\left(M^{-1}(J)\right)$. By simple calculation, we see $I \subseteq M^{-1}(M(I))$ and $M\left(M^{-1}(J)\right) \subseteq J$. We answer the question for the case of ideals.

In general, we have the following result.

THEOREM 2.4. If $S$ is a subset of $X$ with 0 , then $S=M^{-1}(M(S))$.

Proof. Let $x$ be any element of $S$. It follows from the definition of $M$ that $x^{-1} \in$ $M(S)$ and that $x \in M^{-1}(M(S))$, which implies that $S \subseteq M^{-1}(M(S))$. Thus we have $M(S) \subseteq M\left(M^{-1}(M(S))\right)$.

Suppose that $S$ is a subset of $X$ with 0 . It follows that $M(S)$ is a subsemigroup. In fact, we get $0^{-1} \in M(S)$ by $0 \in S$. For any element $\alpha=a_{1}^{-1} \cdots a_{n}^{-1}$ and $\beta=b_{1}^{-1} \cdots b_{m}^{-1}$ $\left(a_{i}, b_{j} \in S(1 \leq i \leq n, 1 \leq j \leq m)\right)$, we have $\alpha \cdot \beta=a_{1}^{-1} \cdots a_{n}^{-1} \cdot b_{1}^{-1} \cdots b_{m}^{-1} \in M(S)$. This means that $M(S)$ is the subsemigroup. If $x \in M\left(M^{-1}(M(S))\right)$, then there exist $a_{i} \in M^{-1}(M(S))(1 \leq i \leq n)$ such that $x=a_{1}^{-1} \cdots a_{n}^{-1}$. Since $a_{i}^{-1} \in M(S)$ and $M(S)$ is the subsemigroup, it follows from $x=a_{1}^{-1} \cdots a_{n}^{-1} \in M(S)$ that $M\left(M^{-1}(M(S))\right) \subseteq$ $M(S)$. These imply that $M(S)=M\left(M^{-1}(M(S))\right)$ and $S=M^{-1}(M(S))$ by Proposition 2.2.

COROLlary 2.5. If $I$ is an ideal, then $I=M^{-1}(M(I))$.

3. Relations between order ideals $J$ and $M\left(M^{-1}(J)\right)$. We proceed to investigate relations between order ideals $J$ and $M\left(M^{-1}(J)\right)$. In general, we have $M\left(M^{-1}(J)\right) \subseteq J$ for any order ideal $J$. Now the following question arises:

Are always $J=M\left(M^{-1}(J)\right)$ ? or Under what condition do we get $J=M\left(M^{-1}(J)\right)$ ?

THEOREM 3.1. For any order ideal $J, M\left(M^{-1}(J)\right)$ is the order ideal generated by $J \cap R(X)$.

Proof. It is clear that $M\left(M^{-1}(J)\right)$ is an order ideal and $J \cap R(X) \subseteq M\left(M^{-1}(J)\right)$.

To establish a proof of the theorem, we only have to show that $M\left(M^{-1}(J)\right) \subseteq K$ for any order ideal $K$ containing $J \cap R(X)$. If $x \in M\left(M^{-1}(J)\right)$ then there are elements $a_{i} \in M^{-1}(J)$ such that $x=a_{1}^{-1} \cdots a_{n}^{-1}$. From $a_{i}^{-1} \in J$, we have $a_{i}^{-1} \in J \cap R(X) \subseteq K$. Since $K$ is an order ideal, we also have $x=a_{1}^{-1} \cdots a_{n}^{-1} \in K$ and hence $M\left(M^{-1}(J)\right) \subseteq K$.

We denote by ( $T]$ the order ideal generated by $T \subseteq M(X)$.

COROLLARY 3.2. For any BCI-algebra $X, M(X)$ is the order ideal generated by $R(X)$, that is, $M(X)=(R(X)]$.

To ask whether $J=M\left(M^{-1}(J)\right)$ is equivalent to do $J=(J \cap R(X)]$ for every order ideal $J$. As the next example shows, we cannot in general conclude $J=M\left(M^{-1}(J)\right)$. 
EXAMPLE 3.3 . Let $X=\{0,1,2, \ldots, a\}$ and let $*$ be an operation defined as in the following table:

\begin{tabular}{c|cccccc}
\hline 0 & 0 & 1 & 2 & 3 & $\cdots$ & $a$ \\
\hline 0 & 0 & 0 & 0 & 0 & $\cdots$ & $a$ \\
1 & 1 & 0 & 0 & 0 & $\cdots$ & $a$ \\
2 & 2 & 1 & 0 & 0 & $\cdots$ & $a$ \\
3 & 3 & 2 & 1 & 0 & $\cdots$ & $a$ \\
$\vdots$ & $\vdots$ & $\vdots$ & $\vdots$ & $\vdots$ & $\ddots$ & $\vdots$ \\
$a$ & $a$ & $a$ & $a$ & $a$ & $\cdots$ & 0 \\
\hline
\end{tabular}

It turns out that $X$ is a BCI-algebra and both $J=\left\{0^{-1}, 1^{-1}, 2^{-1}, \ldots, a^{-1} a^{-1}\right\}$ and $J \cap$ $R(X)=\left\{0^{-1}, 1^{-1}, 2^{-1}, \ldots\right\}$ are order ideals. This means that the order ideal $M\left(M^{-1}(J)\right)$ generated by $J \cap R(X)$ is identical with $J \cap R(X)$ itself. Hence we have $J \neq M\left(M^{-1}(J)\right)$.

In spite of the above, in case of BCK-algebras, we can show $J=M\left(M^{-1}(J)\right)$. It is proved in [4] that $M^{-1}$ is an injection in case of $X$ being a finite $B C K$-algebra. We can prove the same result for any BCK-algebra without the assumption being finite.

THEOREM 3.4. Let $X$ be a BCK-algebra and $J$ an order ideal. Then $J=M\left(M^{-1}(J)\right)$.

Proof. It suffices to prove that $J \subseteq M\left(M^{-1}(J)\right)$. Suppose that $x \in J(\subseteq M(X))$. There exist $a_{i} \in X$ such that $x=a_{1}^{-1} \cdots a_{n}^{-1}$. Since $X$ is a BCK-algebra, we have $a_{i}^{-1}$ 드 $a_{1}^{-1} \cdots a_{n}^{-1}=x \in J$ for each $i$. From $a_{i}^{-1} \in J$ and $a_{i} \in M^{-1}(J)$, we conclude that $x=$ $a_{i}^{-1} \cdots a_{n}^{-1} \in M\left(M^{-1}(J)\right)$. This implies that $J \subseteq M\left(M^{-1}(J)\right)$, that is, $J=M\left(M^{-1}(J)\right)$.

COROLLARY 3.5. For any BCK-algebra $X, I$ is an ideal if and only if $M(I)$ is an order ideal.

Proof. It follows from Proposition 2.2 that if $I$ is an ideal then $M(I)$ is an order ideal. Conversely, let $M(I)$ be any order ideal. Since $X$ is a $B C K$-algebra, we have $M(I)=$ $M\left(M^{-1}(M(I))\right)$ from Theorem 3.4. This implies $I=M^{-1}(M(I))$ because the operator $M$ is injective. It follows that $I$ is the ideal of $X$.

THEOREM 3.6. Let $X$ be a BCI-algebra. Then $M(X)=R(X)$ implies $J=M\left(M^{-1}(J)\right)$ for any order ideal $J$.

Proof. We only show $J \subseteq M\left(M^{-1}(J)\right)$. If $x \in J$ then there exist $a_{i} \in X$ such that $x=a_{1}^{-1} \cdots a_{n}^{-1}$. It follows from the assumption that $x=a_{1}^{-1} \cdots a_{n}^{-1}=b^{-1}$ for some $b \in X$. Since $x \in J$, we have $b^{-1} \in J$ and hence $x=b^{-1} \in M\left(M^{-1}(J)\right)$ by $b \in M^{-1}(J)$. This means that $J \subseteq M\left(M^{-1}(J)\right)$.

REMARK 3.7. The converses of Theorems 3.4 and 3.6 do not hold as in the next example.

EXAMPLE 3.8. For $X=\{0, a, b, c\}$, we define an operation $*$ as in the following table:

\begin{tabular}{c|cccc}
\hline$*$ & 0 & $a$ & $b$ & $c$ \\
\hline 0 & 0 & 0 & 0 & $c$ \\
$a$ & $a$ & 0 & $a$ & $c$ \\
$b$ & $b$ & $b$ & 0 & $c$ \\
$c$ & $c$ & $c$ & $c$ & 0 \\
\hline
\end{tabular}


It is clear that $X$ is not a BCK-algebra but a BCI-algebra. In this case we also have $M(X)=\left\{0^{-1}, a^{-1}, b^{-1}, c^{-1}, c^{-1} c^{-1}\right\}$ and $R(X)=\left\{0^{-1}, a^{-1}, b^{-1}, c^{-1}\right\}$, that is, $M(X) \neq$ $R(X)$. But it turns out that $J=M\left(M^{-1}(J)\right)$ for every order ideal $J$ of $X$.

4. Relations between $I$ and $M(I), J$ and $M^{-1}(J)$. Two maps $M$ and $M^{-1}$ are both monotone. While $I=M^{-1}(M(I))$ for any ideal $I$, we have in general $J \neq M\left(M^{-1}(J)\right)$. This means that $M$ and $M^{-1}$ are not converse maps to each other. We also see that a map $M$ whose domain is restricted to the set of all ideals is an injection, but we do not know about $M^{-1}$ until now. Moreover there is a following problem left open in [4]: let $X$ be a BCI-algebra and $M(X)$ be an adjoint semigroup.

(a) Is a map $I \rightarrow M(I)$ an injection?

(b) Is there a one-to-one correspondence between the set $\Phi(X)$ of all ideals and the set $O(X)$ of all order ideals?

In the following, we give affirmative answers to the questions above.

Proposition 4.1. For every $S \subseteq X$, if $M^{-1}(M(S))$ is an ideal then $M^{-1}(M(S))$ is the ideal generated by $S$.

Proof. We only show that $M^{-1}(M(S)) \subseteq I$ for any ideal $I$ such that $S \subseteq I$. Suppose that $x \in M^{-1}(M(S))$. Since $x^{-1} \in M(S)$, there exist $s_{i} \in S$ such that $x^{-1}=s_{1}^{-1} \cdots s_{n}^{-1}$. It follows that $\left(\cdots\left(x * s_{1}\right) * \cdots\right) * s_{n}=0 \in I$ and hence $x \in I$ from $s_{i} \in S \subseteq I$. This yields the desired result.

Proposition 4.2. If $I$ is an ideal then $M(I)$ is an order ideal generated by $\left\{a^{-1} \mid\right.$ $a \in I\}$.

Proof. It is clear from Proposition 2.2 that $M(I)$ is an order ideal and $\left\{a^{-1} \mid a \in\right.$ $I\} \subseteq M(I)$. Let $J$ be an order ideal of $M(X)$ and $\left\{a^{-1} \mid a \in I\right\} \subseteq J$. If $x \in M(I)$ then there exist $a_{i} \in I$ such that $x=a_{1}^{-1} \cdots a_{n}^{-1}$. It follows from $a_{i} \in I$ that $a_{i}^{-1} \in\left\{a^{-1} \mid\right.$ $a \in I\} \subseteq J$. From supposition, $J$ is the order ideal and of course it is a subsemigroup. Since $a_{i}^{-1} \in J$, we get that $x=a_{1}^{-1} \cdots a_{n}^{-1} \in J$. This implies $M(I) \subseteq J$ and hence $M(I)$ is the order ideal generated by $\left\{a^{-1} \mid a \in I\right\}$.

Proposition 4.3. For any subset $S \subseteq X, M^{-1}(M(S))$ is an ideal if and only if $M(S)$ is an order ideal.

Proof. Suppose that $M^{-1}(M(S))$ is an ideal. It is obvious from the definition that $M\left(M^{-1}(M(S))\right) \subseteq M(S)$. Since $M^{-1}(M(S))$ is an ideal, it is generated by $S$ from the above, so we have $S \subseteq M^{-1}(M(S))$. This means $M(S) \subseteq M\left(M^{-1}(M(S))\right)$ and consequently $M(S)=M\left(M^{-1}(M(S))\right)$, that is, $M(S)$ is the order ideal by Proposition 2.2.

If $M(S)$ is an order ideal, then $M^{-1}(M(S))$ is an ideal from Proposition 2.3.

THEOREM 4.4. If $J$ is a subsemigroup of $M(X)$, then

(1) $M^{-1}\left(M\left(M^{-1}(J)\right)\right)=M^{-1}(J)$,

(2) $M\left(M^{-1}(J)\right)$ is an order ideal if and only if $M^{-1}(J)$ is an ideal.

Proof. (1) It is clear from assumption of the theorem that $M\left(M^{-1}(J)\right) \subseteq J$ and hence $M^{-1}\left(M\left(M^{-1}(J)\right)\right) \subseteq M^{-1}(J)$. On the other hand, it follows from $M^{-1}(J) \subseteq X$ that $x \in M^{-1}(J)$ implies $x^{-1} \in M\left(M^{-1}(J)\right)$ and $x \in M^{-1}\left(M\left(M^{-1}(J)\right)\right)$. Thus we have $M^{-1}(J) \subseteq M^{-1}\left(M\left(M^{-1}(J)\right)\right)$ and $M^{-1}\left(M\left(M^{-1}(J)\right)\right)=M^{-1}(J)$. 
(2) In case of $M\left(M^{-1}(J)\right)$ being an order ideal, since $M^{-1}\left(M\left(M^{-1}(J)\right)\right)$ is an ideal, $M^{-1}(J)$ is the ideal by the above. The converse is clear.

Proposition 4.5. If $I$ is an ideal, then $x \in I$ if and only if $x^{-1} \in M(I)$.

Proof. Suppose that $x^{-1} \in M(I)$. There exist $a_{i} \in I$ such that $x^{-1}=a_{1}^{-1} \cdots a_{n}^{-1}$. Since $\left(\cdots\left(x * a_{1}\right) * \cdots\right) * a_{n}=0 \in I$, we get $x \in I$. The reverse case is clear.

A subset $S$ of $X$ is called closed if $S=M^{-1}(M(S))$. It is clear from the definition of closedness that $S$ is a closed set if and only if the condition holds: $x^{-1} \in M(S)$ if and only if $x \in S$.

From the above we have a relationship between an ideal $I$ and an order ideal $M(I)$.

Proposition 4.6. For any subset I, I is an ideal if and only if $I$ is a closed set and $M(I)$ is an order ideal.

By Proposition 2.2, $M$ is an injection. For $M^{-1}$, we have the following proposition.

Proposition 4.7. For any map $M, M^{-1}$ is an injection if and only if $J=M\left(M^{-1}(J)\right)$ for any order ideal $J$.

Proof. From Theorem 4.4, we have $M^{-1}\left(M\left(M^{-1}(J)\right)\right)=M^{-1}(J)$ for any order ideal $J$. Since $M^{-1}$ is injective, we have $J=M\left(M^{-1}(J)\right)$.

Suppose that $M^{-1}(J)=M^{-1}(K)$ for two order ideals $J, K$. We obtain $J=M\left(M^{-1}(J)\right)$ $=M\left(M^{-1}(K)\right)=K$ and hence $M^{-1}$ is injective.

Thus, from Theorem 3.4, we have

$$
X \text { : BCK-algebra } \Longrightarrow M^{-1} \text { : injection. }
$$

This generalizes the following result in [2] (which unfortunately has a gap in its proof):

$$
X \text { : finite BCK-algebra } \Longrightarrow M^{-1} \text { : injection. }
$$

Also we have from Proposition 2.2 and Theorem 3.6 that for $X$ being a BCI-algebra and each ideal $I, I^{\prime}$,

$$
\begin{gathered}
I \neq I^{\prime} \Longrightarrow M(I) \neq M\left(I^{\prime}\right), \\
M(X)=R(X) \Longrightarrow M^{-1}: \text { injection. }
\end{gathered}
$$

This gives affirmative answers to the Open Problem in [4] which says that: let $X$ be a BCI-algebra and $M(X)$ an adjoint semigroup of it. Then

(a) Is a map $I \rightarrow M(I)$ injective?

(b) If $M(X)=R(X)$, then is there a one-to-one correspondence from the set of all ideals of $X$ to the set of all order ideals of $M(X)$ ?

Because as to (b) if $M(X)=R(X)$ then $M^{-1}$ is injective and it follows from Theorem 3.6 that $J=M\left(M^{-1}(J)\right)$ for every order ideal $J$. This implies that $M$ and $M^{-1}$ are converse maps to each other and hence there is a one-to-one correspondence from $\mathscr{I}(X)$ to $\mathcal{O}(X)$. 


\section{Characterization of some BCI-algebras by $R(X)$ and $M(X)$}

Proposition 5.1. For any BCI-algebra $X, R(X)$ satisfies the condition that for any $a^{-1} \in R(X)$ there is $b^{-1} \in R(X)$ such that $a^{-1} b^{-1}=0^{-1}$ if and only if $X$ is $p$-semisimple, that is, $0 *(0 * a)=a$ for every $a \in X$.

Proof. Suppose that $R(X)$ satisfies the condition that for for each $a^{-1}$ there exists $b^{-1} \in R(X)\left(a^{-1} b^{-1}=0^{-1}\right)$. This implies $(u * a) * b=0$ for every $u \in X$ and hence $0 * a=b$ by taking $u=b$. We thus have $(u * a) *(0 * a)=u$ and $0 *(0 * a)=a$ by taking $u=a$. This means that $X$ is a $p$-semisimple BCI-algebra.

It is easy to prove the converse by taking $b=0 * a$. For, if we take $b=0 * a$ then it suffices to show that $(x * a) * b=x$ for every $x \in X$. It is clear from the following

$$
\begin{aligned}
(x * a) *(0 * a) & =0 *(0 *((x * a) *(0 * a))) \\
& =0 *((0 *(x * a)) *(0 *(0 * a))) \\
& =0 *(((0 * x) *(0 * a)) * a) \\
& =0 *(((0 *(0 * a)) * x) * a) \\
& =0 *((a * x) * a) \\
& =0 *((a * a) * x) \\
& =0 *(0 * x) \\
& =x .
\end{aligned}
$$

If $R(X)$ satisfies the condition that for any $a^{-1} \in R(X)$ there exists $b^{-1} \in R(X)$ such that $a^{-1} b^{-1}=0^{-1}$, then $M(X)$ becomes an abelian group.

COROLLARY 5.2. For any BCI-algebra $X$, if $X$ is $p$-semisimple then $M(X)$ is an abelian group.

In [2], positive implicative BCK-algebras are characterized by $R(X)$. We also give a characterization of those as follows.

Proposition 5.3. The following conditions are equivalent to each other:

(1) for every BCI-algebra $X, R(X)$ is idempotent (i.e, for all $a^{-1} \in R(X) a^{-1} a^{-1}=$ $a^{-1}$,

(2) every BCI-algebra $X$ is a positive implicative BCK-algebra (i.e, a BCK-algebra satisfying the condition: for all $u, a \in X(u * a) * a=u * a)$,

(3) $\left\{u \in M(X) \mid u \sqsubseteq a^{-1}\right\}$ is an order ideal for any $a \in X$.

Proof. It is straightforward from the definition of positive implicativeness that $(1) \Leftrightarrow(2)$.

(1) $\Rightarrow$ (3). Let $\Gamma_{a}=\left\{u \in M(X) \mid u \sqsubseteq a^{-1}\right\}$ and $u=x_{1}^{-1} \cdots x_{n}^{-1}, v=y_{1}^{-1} \cdots y_{m}^{-1}$ be in $\Gamma_{a}$. Since $u v=x_{1}^{-1} \cdots x_{n}^{-1} \cdot y_{1}^{-1} \cdots y_{m}^{-1} \sqsubseteq a^{-1} a^{-1}=a^{-1}, \Gamma_{a}$ is a subsemigroup of $M(X)$. If $u=x_{1}^{-1} \cdots x_{n}^{-1} \in \Gamma_{a}$ and $b^{-1} \sqsubseteq u$, then we have $b^{-1} \sqsubseteq a^{-1}$ from $b^{-1} \sqsubseteq u \sqsubseteq a^{-1}$. It follows from $b^{-1} \in \Gamma_{a}$ that $\Gamma_{a}$ is an order ideal.

(3) $\Rightarrow(1)$. If $\Gamma_{a}$ is an order ideal, then it is also a subsemigroup. Thus, we have $a^{-1} a^{-1} \in$ $\Gamma_{a}$ from $a^{-1} \in \Gamma_{a}$, that is, $a^{-1} a^{-1} \sqsubseteq a^{-1}$. This yields $u * a \leq(u * a) * a$ for any $u \in X$. If we take $u=a$, then we have $0 \leq 0 * a$. It follows from $0 *(0 * a) \leq a$ that $0 \leq a$. 
In this case we also have $((u * a) * a) *(u * a)=0 * a=0,(u * a) * a \leq u * a$ and $(u * a) * a=u * a$. This means $a^{-1} a^{-1}=a^{-1}$ and hence $R(X)$ is idempotent.

\section{REFERENCES}

[1] W. Huang, On the semigroup theory of BCI-algebras, Pure Appl. Math. 9 (1993), 28-34.

[2] W. Huang and D. Wang, Adjoint semigroups of BCI-algebras, Southeast Asian Bull. Math. 19 (1995), no. 3, 95-98. MR 96h:06039. Zbl 0859.06016.

[3] M. Kondo, Some properties of left maps in BCK-algebras, Math. Japon. 36 (1991), no. 1, 173-174. CMP 1093 369. Zbl 0717.06011.

[4] J. Li, Relationship between filters of BCK-algebra and order ideals of its adjoint semigroup, Math. Japon. 49 (1999), no. 3, 447-450. MR 2000d:06028. Zbl 0932.06016.

Michiro Kondo: DePARTMENT OF MATHEMATICS AND COMPUTER SCIENCE, Shimane UNIVERSITY, MATSUE, 690-8504, JAPAN

E-mail address: kondo@cis.shimane-u.ac.jp 


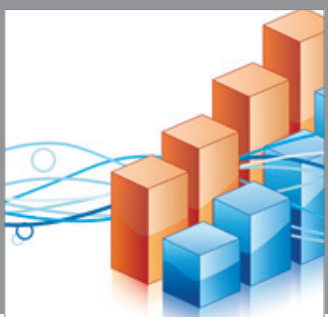

Advances in

Operations Research

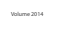

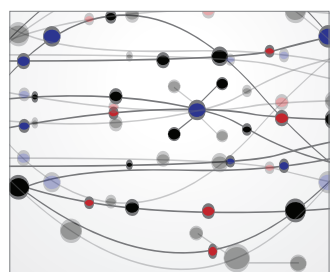

\section{The Scientific} World Journal
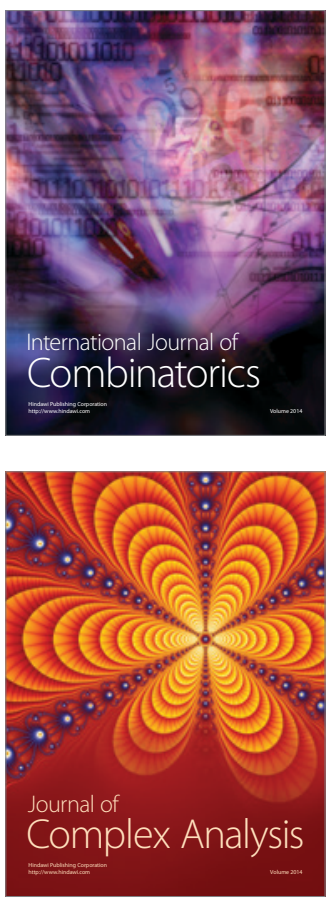

International Journal of

Mathematics and

Mathematical

Sciences
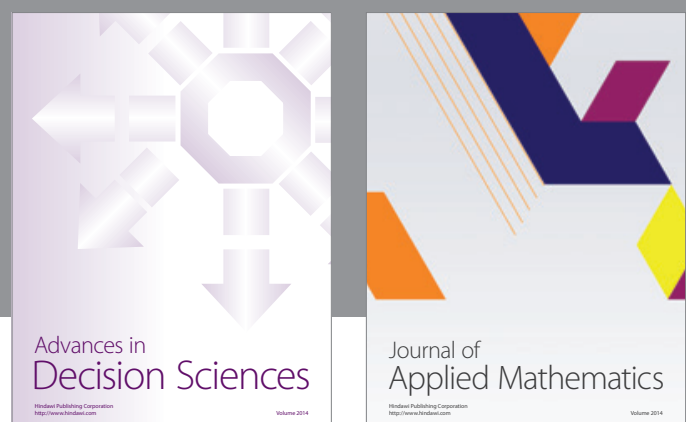

Journal of

Applied Mathematics
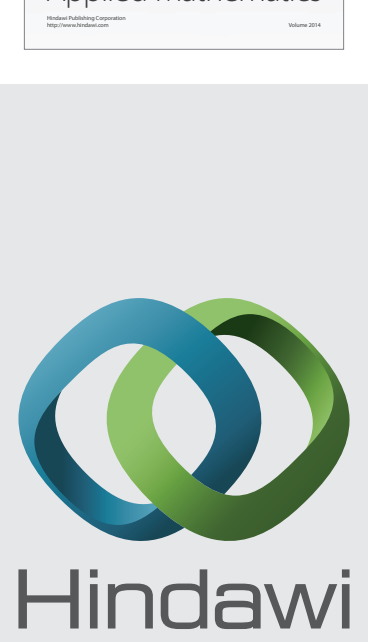

Submit your manuscripts at http://www.hindawi.com
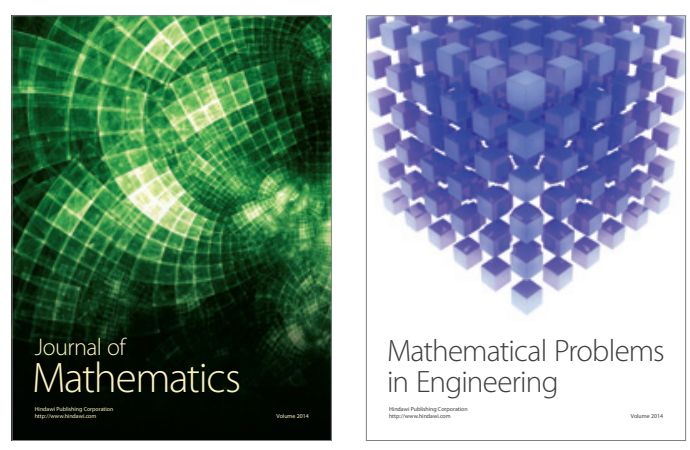

Mathematical Problems in Engineering
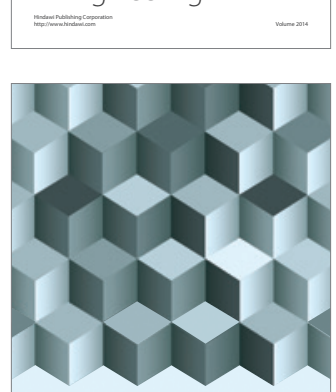

Journal of

Function Spaces
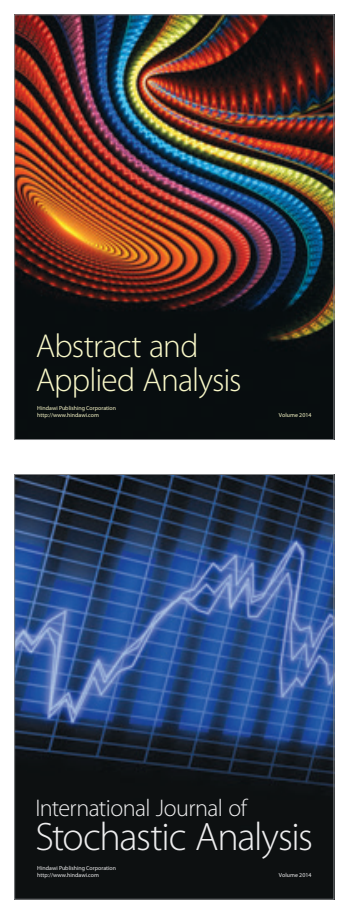

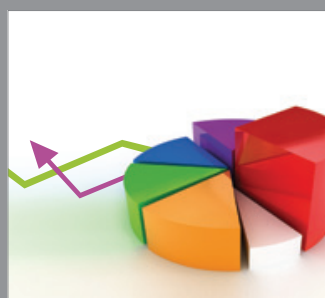

ournal of

Probability and Statistics

Promensencen
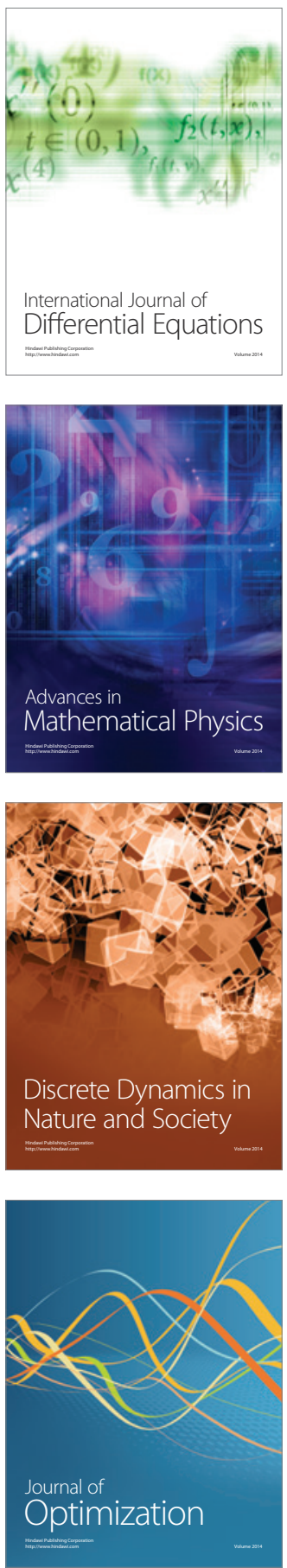